\section{The Unified Protocol for Transdiagnostic Treatment of Perinatal Depression: A Case Study}

Clinical Case Studies 2020, Vol. 19(6) 49I-508 (C) The Author(s) 2020

Article reuse guidelines: sagepub.com/journals-permissions DOI: 10.1 I 77/I534650I2095I266 journals.sagepub.com/home/ccs

\author{
Elena Crespo-Delgado ${ }^{1,2}$, Verónica Martínez-Borba ${ }^{1,2}$, \\ and Jorge Osma ${ }^{2,3}$
}

\begin{abstract}
This case study aims to present the application of the Unified Protocol (UP) for the Transdiagnostic Treatment of Emotional Disorders in the case of a 38-year-old pregnant woman with a peripartum onset of major depressive disorder and anxiety symptoms. The UP is a cognitive-behavioral intervention based on the shared etiological and maintenance mechanisms of emotional disorders (i.e., mood, anxiety, and related disorders). The UP helps to modify maladaptive emotion-regulation strategies and enhance tolerance of unpleasant and intense emotions (sadness, anxiety, anger, etc.). Whereas previous studies demonstrate the efficacy of the UP in different emotional disorders, to our knowledge, none of them have used the UP to treat perinatal emotional disorders. After the eight UP modules of individual treatment, a significant decrease in anxiety, depression, negative affect, and anxiety sensitivity, and also an increase in positive affect were observed. These results were maintained at the 12-month follow-up. In addition to its clinical utility and acceptance, these results indicate that the UP can be adapted and personalized for the treatment of perinatal emotional disorders. Future studies with larger samples and controlled experimental designs should be developed to demonstrate its efficacy. Furthermore, as the UP seems to be useful in a group format, future efforts should be focused on the UP with perinatal women in a group format.
\end{abstract}

\title{
Keywords
}

transdiagnostic, unified protocol, perinatal, postpartum depression, emotion regulation

\section{Theoretical and Research Basis for Treatment}

Both pregnancy and postpartum are vital stages in which women face various changes and challenges that sometimes result in emotional disorders. Among all the emotional disturbances, perinatal depression, currently understood as the depressive symptomatology that takes place during pregnancy or up to 1 year postpartum (Hamel et al., 2019), stands out. Perinatal depression is a highly under-diagnosed and under-treated disorder (Cox et al., 2016), hindering accurate estimates in terms of prevalence. Global data indicate that $10 \%$ to $13 \%$ of women suffer from some

\footnotetext{
'Universitat Jaume I, Castellón, Spain

${ }^{2}$ Instituto de Investigación Sanitaria de Aragón, Zaragoza, Spain

${ }^{3}$ Universidad de Zaragoza, Teruel, Spain

\section{Corresponding Author:}

Jorge Osma, Departamento de Psicología y Sociología, Facultad de Ciencias Sociales y Humanas, Universidad de Zaragoza, C/Ciudad Escolar s/n., 44003 Teruel, Spain.

Email: osma@unizar.es
} 
mental disorder during pregnancy or postpartum, with perinatal depression predominating (World Health Organization, 2019). Also, from a clinical point of view, it must be considered that half of the women with depression also present anxious symptoms (Dennis et al., 2017).

Perinatal depression produces negative consequences for the mother and the fetus/baby and society, including the risk of suicide or infanticide, preeclampsia, increased maternal use of substances, birth complications, premature delivery, low birth weight, and difficulties in the child's socio-emotional, behavioral, and cognitive development (Lobel \& Dunkel-Schetter, 2016). All this implies a high personal and family suffering in addition to a significant economic expense, derived both from the use of health resources and the loss of productivity (Danaher et al., 2013).

Fortunately, we currently have effective psychological interventions to address depressive symptoms in the perinatal stage. Some examples are interventions based on cognitive-behavioral therapy (e.g., Milgrom et al., 2015), interpersonal therapy (e.g., Sockol, 2018), or social support (e.g., Dennis, 2014). All these interventions are characterized by using a common procedure, the application of a specific treatment (e.g., cognitive-behavioral therapy) to a specific diagnosis (e.g., major peripartum depressive disorder). This characteristic approach to categorical psychopathological and clinical models has important limitations in routine clinical practice. First, because treating specific disorders does not address problems classified as "unspecified" (Brown et al., 2001). Second, because high comorbidity between different emotional disturbances (Barlow et al., 2013) leads clinicians either to treat the main pathology and expect the effects of treatment to affect the comorbid pathology, or to apply two treatments simultaneously (McManus et al., 2010). This involves a large consumption of resources, both personal and economic (Barlow et al., 2004). Additionally, from the perspective of implementation and dissemination of evidence-based psychological treatments, this approach is very expensive in terms of the education and training of therapists, as they must learn each of the specific treatments developed for each of the mental disorders (Barlow et al., 2004).

As a solution to these limitations, different transdiagnostic treatments have recently been designed to address Emotional Disorders (ED; mood, anxiety, and related disorders; Bullis et al., 2019). One of them is the Unified Protocol (hereinafter UP; Barlow et al., 2004), which postulates the existence of shared etiological and maintenance mechanisms among the different ED. Some of these mechanisms are the personality dimensions of high neuroticism and low extraversion, anxiety sensitivity, intolerance of uncertainty, avoidance behaviors, and rumination, among others (Sherman et al., 2018). People with ED experience frequent and intense emotions that cause them discomfort, leading to an aversive reaction to emotions and the initiation of emotional avoidance behaviors that, in the short term, will bring relief but, in the long term, will maintain the emotional distress (Bullis et al., 2019). The UP aims to address these shared etiological and maintenance mechanisms, that is, to train in new adaptive emotion regulation strategies to tolerate intense emotions and improve patients' quality of life (Barlow et al., 2018a).

The UP has been applied in different EDs with positive results (Barlow et al., 2017), recently considering the need to transfer these transdiagnostic treatments to the approach of perinatal anxiety and depression (Pilkington et al., 2015). The clinical utility and feasibility of the UP for the treatment of perinatal depression has not been studied so far, so the aims of the present study are: (a) to present a case of perinatal depression in which the UP was applied, (b) to describe the adaptations of the UP that were made to adjust the intervention to the particularities of perinatal depression, and (c) to present the main results obtained by the different treatment sessions. We expected to find an improvement in the clinical symptoms after the application of the UP, maintenance of the improvement at the long term (12-month follow-up) as well as the patient's high satisfaction with the intervention.

\section{Case Introduction}

Patricia (pseudonym) is a 38 -year-old woman, married for 18 years, with a 6-year-old son. She was in the 34 th week of her second pregnancy when she came to our clinic for treatment. She has 
been living in a rural area of about 7,000 inhabitants for 11 years. Her income is currently low, as she is on sick leave, and her husband is a self-employed house painter and only works when someone hires him, which is not very often, as they live in a small town. The relationship with the in-laws is not good, but the relationship with her own family is good, although they do not provide much support, despite living in the same locality. The therapist who performed the intervention (E.C.) is a graduated psychologist, holds a Master's Degree in Psychology, and has more than 20 years' clinical experience. She has also been trained in Cognitive Behavioral Therapy and in the application of the UP. During the intervention, the therapist was supervised by J.O., certified by the Unified Protocol Institute as a trainer and researcher in the UP.

\section{Presenting Complaints}

At the time of her consultation, Patricia presented a Major Depressive Disorder (MDD) with peripartum specifier and obsessive personality traits according to the Diagnostic and Statistical Manual of Mental Disorders-text revision (5th ed.) (American Psychiatric Association [APA], 2013). The symptoms reported by Patricia were sadness, anhedonia, daily crying, fatigue, abulia (i.e., lack of motivation and energy in daily activities), insomnia, feelings of guilt and worthlessness, and concentration problems. She was on sick leave and did not have a permanent post and her husband was self-employed with a low income. She has a bad relationship with the in-laws and regular couple arguments. She refers to the context in which she lives, a small inland town, as a "village culture," in which practically the whole neighborhood knows all about their neighbors' lives, and she does not want to live there, but she has difficulty making decisions, and her husband does not want to move to a larger town.

\section{History}

Her mother was diagnosed with depression more than 20 years ago, and a paternal aunt with "severe depression" died of autolysis. Patricia was diagnosed with MDD at age 18 and took medication for more than 5 years. Her first pregnancy was normal, both physically and emotionally. At a physical level, the current pregnancy is going smoothly, and there are no medical conditions of concern. As for emotional well-being, she has been in a low mood since the 20th week of pregnancy, when it was confirmed that the baby she was expecting was a boy, when she wanted a girl. Her mood worsened around the 30th week of gestation when she noticed, in addition to low mood, unspecified anxiety symptoms (she did not meet the criteria for any disorder). The patient expressed negative thoughts and concerns such as: "I won't be able to take care of both children," "the ultrasound will go wrong," "I won't know how to act with the baby," or "I can not make decisions."

\section{Assessment}

Prior to treatment, three evaluation sessions were conducted. During the first session, the informed consent was filled in, and a screening interview was administered to determine the sociodemographic data and the reason for the consultation. For the first screening of ED (especially anxiety and mood), the Mini Neuropsychiatric International Interview (MINI; Sheehan, 2015) was administered. At the end of this session, Patricia was given the following set of questionnaires to complete at home and bring back to the next session:

Overall Anxiety Severity and Impairment Scale (OASIS; Norman et al., 2006; Osma et al., 2019). This evaluates frequency, intensity, and interference of anxious symptomatology in the person's life over the last week. It consists of five items that are rated on a Likert scale ranging from 0 (I didn't feel anxious) to 4 (Constant anxiety). The total score is obtained from the sum of all the items and ranges from 0 to 20 . Higher scores indicate greater severity of the anxious symptomatology. In the Spanish version, the cut-off point established in the clinical sample was 10 points (Osma et al., 2019). The OASIS has good internal consistency ( $\alpha=.87$; Osma et al., 2019). 
Overall Depression Severity and Impairment Scale (ODSIS; Bentley et al., 2014; Osma et al., 2019). This evaluates frequency, intensity, and interference of sadness/depression in the person's life during the last week. Like the OASIS, it consists of five items with a response ranging between 0 (I didn't feel depressed) and 4 (Constant depression). Total scores, ranging from 0 to 20 , indicate the severity of depressive symptomatology. In the Spanish version, the cut-off point established in the clinical sample was 10 points (Osma et al., 2019). ODSIS has shown excellent psychometric properties ( $\alpha=.94$; Osma et al., 2019).

Edinburgh Postnatal Depression Scale (EPDS; Cox et al., 1987; Garcia-Esteve et al., 2003). This questionnaire evaluates the presence of depressive symptoms, specifically in the postpartum period. It contains 10 items that evaluate the symptomatology of the last 7 days. Three of these items are direct, and the response ranges from 0 (As much as ever) to 3 (No, not at all/never). The other seven items are inverse, ranging from 0 (No, never) to 3 (Yes, most of the time). Total scores range from 0 to 30. Higher scores indicate greater depressive symptomatology. In the Spanish version, the cut-off point established was 10/11 (Garcia-Esteve et al., 2003). This scale has shown good internal consistency ( $\alpha=.84$; Tsai et al., 2013).

Positive and Negative Affect Schedule (PANAS; Watson et al., 1988; Sandín et al., 1999). This instrument evaluates the person's positive affect (PANAS-PA) and negative affect (PANAS-NA) through 10 positive affect and 10 negative affect descriptors. These items are rated on a Likerttype scale ranging from 1 (nothing or almost nothing) to 5 (very much). Higher scores indicate more positive or negative affect. The Spanish version has shown good internal consistency in both the Positive Affect scale $(\alpha=.87)$ and the Negative Affect scale $(\alpha=.89$; Sandín et al., 1999).

Anxiety Sensitivity Inventory-3 (ASI-3; Taylor et al., 2007; Sandín et al., 2007). This instrument contains 18 items that evaluate the physical, cognitive, and social dimensions of anxiety sensitivity. Each subscale consists of six items that are rated on a Likert-type scale ranging from 0 (nothing or almost nothing) to 4 (very much). Higher scores indicate greater sensitivity to anxiety. This questionnaire has shown good internal consistency in its Spanish validation $(\alpha=.91$; Sandín et al., 2007).

Second and third session: to determine the most relevant data that may be influencing the development or maintenance of the problem, a biographical interview was administered in which information was collected on different areas of Patricia's life (family, work, academics, interests and motivations, couple, sexual, social life, parenting, and health).

Progress assessment over the treatment sessions: the OASIS and ODSIS were administered.

Post-treatment: the same battery of instruments as in the first session was administered again: the MINI interview to confirm that she did not meet the criteria for any disorder, the OASIS, ODSIS, EPDS, PANAS, and ASI-3.

Follow-up at 12 months: the OASIS, ODSIS, and EPDS were administered.

\section{Case Conceptualization}

To start the case conceptualization within the framework of the UP and to assess whether the UP is appropriate for Patricia, it is important to identify the extent to which Patricia is experiencing (a) intense unpleasant emotions and (b) aversive reactions to these emotional experiences, and (c) engaging in emotional behaviors.

When facing challenging situations, she experienced intense emotions and aversive reactions (thoughts and physical sensations). Her inadequate emotion-regulation skills to overcome the distress, namely emotional avoidance strategies, produce distress relief in the short term but, in the long term, its avoidance reinforces her negative beliefs and maintains the emotional distress expressed in anxiety and depressive symptoms.

The problem presented by Patricia during the initial assessment sessions was difficulty in managing negative emotions in diverse contexts such as medical procedures related to the baby's health, conversations with her partner, or meetings with her in-laws. She also presented constant 
concerns about the care of her two children and difficulty making decisions in everyday life. The intense unpleasant emotions reported in these situations were frustration, worthlessness, guilt, anhedonia, lack of motivation and energy, fatigue, anxiety, and continuous sadness. Due to this emotional distress, she experienced both physical and cognitive aversive reactions. For instance, she reported tachycardia and suffocation and thoughts as "They should know how I feel," "I can't do all my duties," or "What if I'm wrong?" As a result, she performed the following emotional avoidance strategies: (a) situation avoidance/escape: not going to meals with the in-laws or avoiding "complicated" conversations with her partner; (b) subtle avoidance behaviors: procrastinating household chores, not bringing up conversation topics with her husband that may end up in an argument; (c) cognitive avoidance: being distracted by watching TV or reading to keep from thinking; (d) safety signals: talking on the mobile phone when she is likely to meet her inlaws in the street. The aforementioned avoidance behaviors reinforced her feelings of frustration, worthlessness, guilt, and sadness and her negative thoughts of incompetence as a mother (e.g., "I won't know how to take care of my two children" or "I'll be a bad mother") resulting in the maintenance of anxiety and depressive symptoms.

The UP is a flexible modular treatment which can be adapted as required to help patients to learn new adaptive emotion-regulation strategies to tolerate intense emotions. It consists of eight modules that are applied over 12 to 16 weekly 1 -hr sessions. Of the eight modules, five are considered active or central because patients train a specific emotion-regulation skill (Modules 3-7). In Patricia's case, the focus of treatment and the distribution of the sessions for each module is shown in Table 1 (core modules are marked in gray).

\section{Course of Treatment and Assessment of Progress}

\section{Course of Treatment}

Patricia came to our clinic in the 34th week of pregnancy, referred by her gynecologist because she presented sadness, anhedonia, lack of motivation and energy, and anxiety symptoms, among others. The treatment took place in 15 1-hr sessions, three for evaluation and rapport building, and 12 for the UP treatment. Throughout the treatment, Patricia used the UP patient workbook (Barlow et al., 2018b) but, given the flexibility of the UP, and to personalize the UP to her perinatal circumstances, we included additional information (e.g., emotional changes during pregnancy and postpartum period, myths about motherhood) and exercises (e.g., mindfulness exercises focused on the baby's movements or baby care, problem-solving) in some of the UP modules, as we will describe in the following section. Below is the procedure description of each UP module:

Module 1. Setting Goals and Maintaining Motivation: As discussed above, the perinatal stage is a time of great change where multiple concerns arise, especially related to the health of the baby (Caparros-Gonzalez et al., 2019). However, these concerns, and therefore the goals of therapy, also evolve depending on whether a woman is pregnant or postpartum when she arrives at our clinic. In perinatal women, goals may vary depending on the women's stage of pregnancy and they may need to be adapted. It is common to include goals related to health and physical well-being during pregnancy (i.e., sleep habits, diet, physical exercise). In the postpartum period, there are usually goals related to improving assertive communication skills, problem-solving, and bonding with the baby. In our case, Patricia was anxious about medical tests for fear that "something would go wrong with the baby," whereas postpartum concerns were more focused on her ability to take care of her children. For this reason, the UP was adapted so that Patricia's goals were set at each stage of the perinatal period, in the short term before delivery (e.g., to regulate her emotional response to anxiety before medical visits), in the medium term after delivery (e.g., to improve her communication style with her partner, using an assertive style), and in the long term after delivery (e.g., to express her habitual feelings and make relevant decisions about where she wants to live). 
Table I. Description of the Modules and Objectives of UP.

\begin{tabular}{|c|c|}
\hline Modules and sessions & Objectives \\
\hline $\begin{array}{l}\text { Module I. (Session I) } \\
\text { Setting Goals and } \\
\text { Maintaining Motivation }\end{array}$ & $\begin{array}{l}\text { 1. Identify problem areas. } \\
\text { 2. Specify objectives to be achieved. } \\
\text { 3. Establish specific steps to solve the problems. } \\
\text { 4. Address the importance of motivation for change. } \\
\text { 5. Explore costs and benefits of changing or staying the same. }\end{array}$ \\
\hline $\begin{array}{l}\text { Module 2. (Sessions } 2 \text { and 3) } \\
\text { Understanding emotions }\end{array}$ & $\begin{array}{l}\text { I. Present the adaptive functionality of emotions, both "positive" } \\
\text { and "negative." } \\
\text { 2. Identify the three components of emotional experience } \\
\text { (thoughts, physical sensations, and behaviors). } \\
\text { 3. Emotional learning. } \\
\text { 4. Analysis of the emotional response (Antecedent, emotional } \\
\text { Response, and short- and long-term Consequences: ARC). } \\
\text { 5. Identify patterns in her emotional triggers (what causes her } \\
\text { emotional experiences) and in the behaviors she engages in. } \\
\text { 6. Introduce the concept of emotion-driven behavior. }\end{array}$ \\
\hline $\begin{array}{l}\text { Module 3. (Sessions } 4 \text { and 5) } \\
\text { Mindful Emotion } \\
\text { Awareness }\end{array}$ & $\begin{array}{l}\text { 1. Identify emotional responses. } \\
\text { 2. Train emotional awareness that is present-centered and } \\
\text { non-judgmental. }\end{array}$ \\
\hline $\begin{array}{l}\text { Module 4. (Sessions } 6 \text { and } 7 \text { ) } \\
\text { Cognitive Flexibility }\end{array}$ & $\begin{array}{l}\text { I. Present the relationship between thought, physical sensations, } \\
\text { and behaviors. } \\
\text { 2. Introduce the concept of automatic evaluation. } \\
\text { 3. Assist in the identification of thinking errors. } \\
\text { 4. Practice cognitive flexibility through cognitive reappraisal. }\end{array}$ \\
\hline $\begin{array}{l}\text { Module 5. (Session 8) } \\
\text { Countering Emotional } \\
\text { Behaviors }\end{array}$ & $\begin{array}{l}\text { I. Introduce emotional behaviors. } \\
\text { 2. Learn the maladaptive role of emotional behaviors. } \\
\text { 3. Identify own strategies of emotional behaviors. } \\
\text { 4. Discuss in detail emotion-driven behaviors, identify them, and } \\
\text { learn alternative trends of action. }\end{array}$ \\
\hline $\begin{array}{l}\text { Module 6. (Session 9) } \\
\text { Understanding and Confronting } \\
\text { Physical Sensations }\end{array}$ & $\begin{array}{l}\text { I. Identify physical sensations associated with emotions. } \\
\text { 2. Understand the role of physical sensations in emotional response. } \\
\text { 3. Expose herself to uncomfortable physical sensations. }\end{array}$ \\
\hline $\begin{array}{l}\text { Module 7. (Sessions I0 and II) } \\
\text { Emotion Exposures }\end{array}$ & $\begin{array}{l}\text { I. Understanding the purpose of emotional exposure. } \\
\text { 2. Establish the hierarchy of emotional and situational avoidance and } \\
\text { practice it. }\end{array}$ \\
\hline $\begin{array}{l}\text { Module 8. (Session I2) } \\
\text { Recognizing Accomplishments } \\
\text { and Looking to the Future }\end{array}$ & $\begin{array}{l}\text { I. Review the skills for coping with emotions learned during the } \\
\text { previous sessions. } \\
\text { 2. Review the evolution during the treatment. } \\
\text { 3. Promote the generalization of skills to other contexts and } \\
\text { encourage motivation for continuous progress. }\end{array}$ \\
\hline
\end{tabular}

Module 2. Understanding emotions: Recognition and analysis of emotional response: In a context as stigmatized as perinatal depression, it is essential to normalize symptoms and provide psychoeducation. During pregnancy, emotions frequently generate discomfort that women do not accept or tolerate because it is not what is "socially" expected of them-society expects them to be happy. In this sense, several authors have highlighted the importance of providing psychoeducation not only for the psychological problem presented by the patient (e.g., depression) but also for the whole process (Donahue et al., 2019), such as motherhood and the myths that surround it (e.g., "it is the most beautiful moment in a woman's life"), the natural emotional changes that usually accompany the pregnant woman throughout this period (e.g., fear and 
anxiety in case something goes wrong before coming to the regular gynecological check-up), and the ambivalence they may experience regarding the pregnancy itself (e.g., "it may affect my career, but how can I think that, if it's my baby and I have to prioritize it"). Therefore, the psychoeducation in this module focused on normalizing concerns about pregnancy and discussing the negative emotions inherent in the myths of motherhood that Patricia presented (e.g., "I'm supposed to feel happy and I don't"). In this sense, the emotional changes that usually occur in the perinatal period, with the habitual ambivalence and cognitive dissonances, were explained. Therefore, what emotions are, their function, their components, and how to analyze and record them in the ARC UP form were all explained to Patricia, and she was thus made aware of her usual responses and the possible existence of maladaptive behavioral patterns. For instance, that week, Patricia registered a family incident that led to an argument among several family members, which caused an increase in her depressive symptoms.

Module 3. Mindful Emotion Awareness: Learning to observe experiences: Three exercises are used in the UP treatment to train emotional awareness as they are described in the UP Therapist Guide (Barlow et al., 2018a, p. 87): (a) focusing on the present without judging through a brief guided "Mindful Emotion-Awareness Meditation" exercise in a neutral mood, so the patient can learn how to anchor in the present without judging; (b) The second exercise is a "Mindful Mood Induction" through music, so they can practice being non-judgmental and present-focused in a controlled emotional context; and (c) "Anchoring in the Present" with step-bystep instructions to practice mindful attention when emotions come up in daily life. Patricia used breathing as a signal to focus and anchor herself in the here and now. She learned to observe her emotions and feelings, identify them, and let them go without judging. Additionally, during pregnancy, extra mindfulness exercises can be performed through sight and touch (of the belly), observing and describing the internal sensations that the baby provokes when moving. In the case of Patricia, as she was pregnant at the time of implementation, she was asked to observe her emotions and her sensations while being fully aware of her baby, to observe how the baby moved inside her, to visualize him and observe his movements. During the postpartum period, these exercises can also be done by observing the baby (with all the senses) at different specific and special moments, such as at bath time or during a massage.

Module 4. Cognitive Flexibility: In pregnancy and postpartum stages, women face multiple stress situations (e.g., typical pregnancy physical discomfort when sleeping; unplanned or unwanted visits after delivery, etc.) that can lead to "thinking traps" (e.g., "I won't know how to care for my two children," "I'll be a bad mother"). In these cases, the patient has to specify the thought, because "not knowing how to care for the baby" could be really terrible for the mother. In these cases, it is necessary to define beforehand what she means by "not knowing how to take care of the baby" or being a "bad mother" in order to carry out the cognitive reappraisal of such negative thoughts appropriately. For this purpose, the UP Downward Arrow strategy can sometimes be useful. In Patricia's case, some of the rigid ideas that caused her distress were her desire to have a baby girl and her trouble when making day-to-day decisions. For this reason, we worked on decatastrophizing the fact of delay when deciding or the baby's sex. We also addressed the reappraisal of the probability that she would not know how to act with the baby or that she would not know how to care for both children.

Enhancement of problem-solving: Patricia's obsessive traits made it difficult for her to make decisions and solve everyday situations, so in Module 4, we included the Problem-Solving Technique (D'Zurilla \& Nezu, 2007) so that she could use it when thoughts such as "I'm not going to have time to do everything I must do" assailed her. This thought was justified if her partner was working that day and could not do part of the housework. Once she learned how to apply the problem-solving technique, the difficulty she encountered was that she did not know when she had to reassess her thoughts (cognitive reappraisal) and when she had to apply the problem-solving technique. These doubts arose after she started to work on this module in the 
following weeks, so Figure 1 was elaborated so she could consult it in moments of doubt and discern when to perform the problem-solving technique and when to perform cognitive reassessment or any other skill learned.

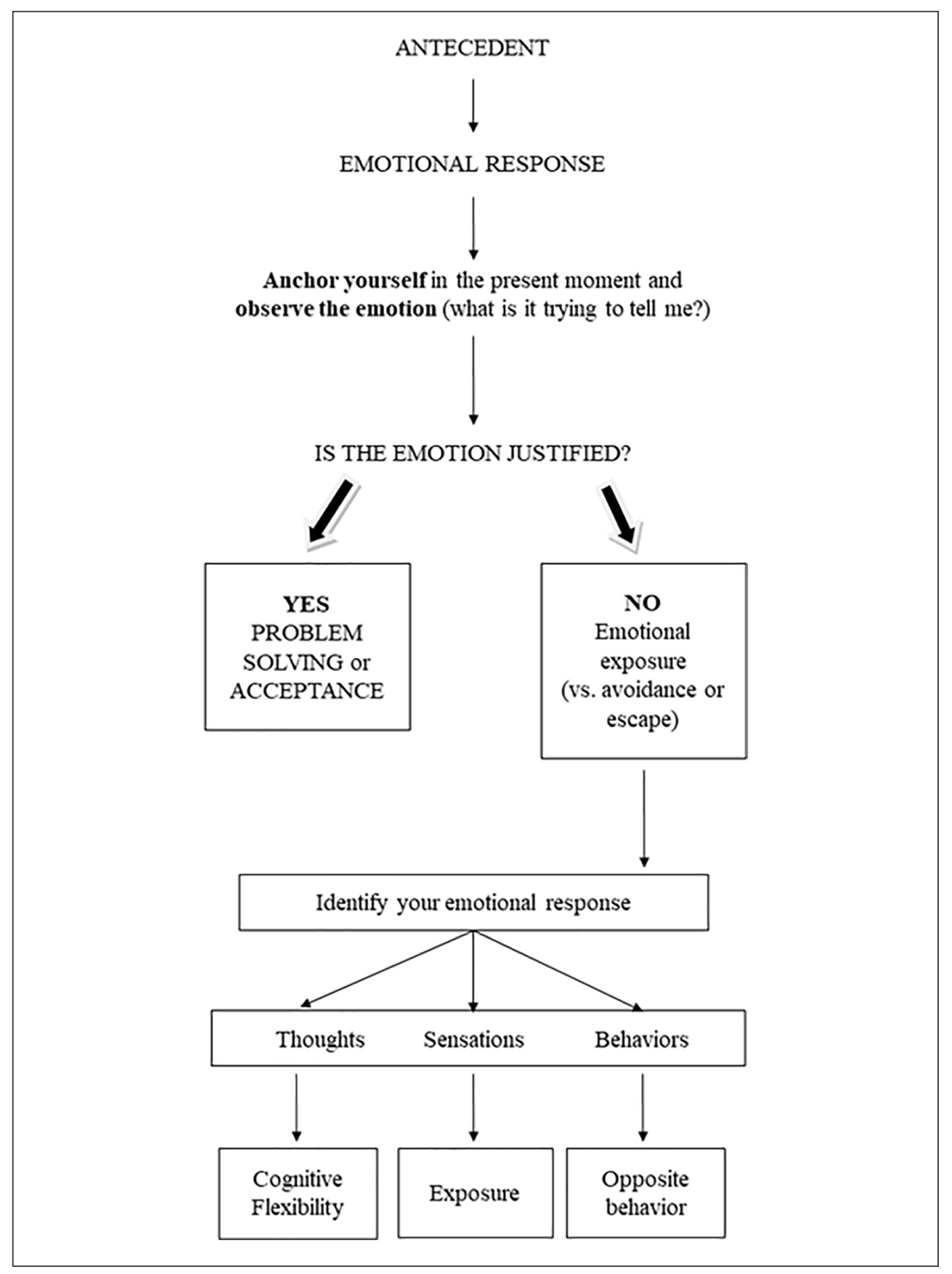

Figure I. Decision diagram. 
Module 5. Countering Emotional Behaviors: Patricia was exposed to situations such as starting conversations with her husband that often ended in arguments, which she avoided because they generated unpleasant emotions. Patricia had to look for alternative behaviors that were incompatible with emotion-driven behaviors, for example, bringing up with her husband a topic of conversation that concerned her.

Sometimes pregnant and postpartum women stop doing enjoyable activities because they feel that the priority is just the baby. The guilt they may feel when they spend time on themselves can reduce their amount of activity and generalize this avoidance to other situations. A goal to work on was to tolerate the emotion of guilt and reassess the thoughts that could appear, through the establishment of pleasant activities (within each woman's possibilities) and to identify avoidance behaviors related to not looking at or holding the baby in the case of severe depressive symptoms and bonding alterations. Pleasant activities were planned with Patricia such as walking by herself for an hour or taking a long bath with no children bothering her.

Module 6. Understanding and Confronting Physical Sensations: Both in pregnancy and postpartum, there may be more physical sensations (e.g., tiredness or sleepiness at any time of the day during pregnancy, or painful sensations due to stitches in the postpartum period). Women with depression may associate these physical sensations with increased feelings of anxiety, sadness, worthlessness, or guilt (e.g., for not being able to do housework because of postpartum pain). It is important to help them consider the physical sensations as temporary, and typical of pregnancy or postpartum while working through the exposure to these sensations. With Patricia, we had to address all these sensations and also the high heartrate that she sometimes presented when, for example, she met her in-laws on the street. To deal with the physical sensations, we practiced exposure by asking her to walk at a fast pace and/or climb the stairs of her house several times.

Module 7. Emotion Exposures: To overcome emotional difficulties related to anxious and depressive symptoms, it is necessary to tolerate intense emotions. This module helps the patient to learn to perform a progressive exposure hierarchy to all situations, objects, thoughts, images and/or sensations that generate discomfort. Now, all the emotion-regulation strategies learned during the treatment are put into practice. Emotional exposure to situations that generate anxiety, guilt, and sadness, such as "setting limits to unexpected visits," "asking for co-responsibility in household tasks," or exposing oneself to "important conversations with one's partner," is common.

We worked with Patricia on exposure to situations such as going to eat at her in-law's house. We used the skills of full emotional awareness without judgment, cognitive flexibility, and alternatives to emotion-driven behaviors. She avoided unpleasant situations, hoping that this would reduce her emotional discomfort but, as it was a maladaptive strategy, instead of eliminating the problem, she maintained it. Patricia gradually exposed herself to all the situations and guilt feelings she noticed when she performed any of these behaviors.

Module 8. Recognizing Accomplishments and Looking to the Future: Treatment may be completed before the postpartum period if it begins during the first trimester of pregnancy. Therefore, at the end of the intervention, in addition to encouraging motivation to maintain achievements, it is important to set new goals for the postpartum period and to think about future situations that may be stressful to develop action plans focused on the postpartum period. We reviewed Patricia's trajectory with her, reinforced the achievements and skills she had learned throughout the intervention. We analyzed what remained to be worked on of the proposed goals both before and after the birth, and Patricia established her working guide to continue being her "own therapist" and keeping moving forward.

\section{Assessment of Progress}

Patricia's assessment of progress during treatment was analyzed through the two main UP instruments (OASIS and ODSIS). As described in the "Assessment" section, these two instruments 
were administered before treatment and once a week to assess progress. Next, we will graphically present the results that show how Patricia evolved throughout the treatment and after its completion in anxiety (OASIS), depression (ODSIS and EPDS), and other clinical variables (PANAS-PA, PANAS-NA, and ASI-3).

Longitudinal evolution by modules of the anxiety and depression variables: Figure 2 shows the evolution of OASIS and ODSIS throughout the eight treatment modules. The evolution of anxious and depressive symptoms decreased throughout the modules. The initial OASIS score was very high in the first module (17 out of a maximum of 20), and this score decreased to 13 toward the middle of the intervention protocol (Module 4) until reaching a score of 4 after finishing Module 8.

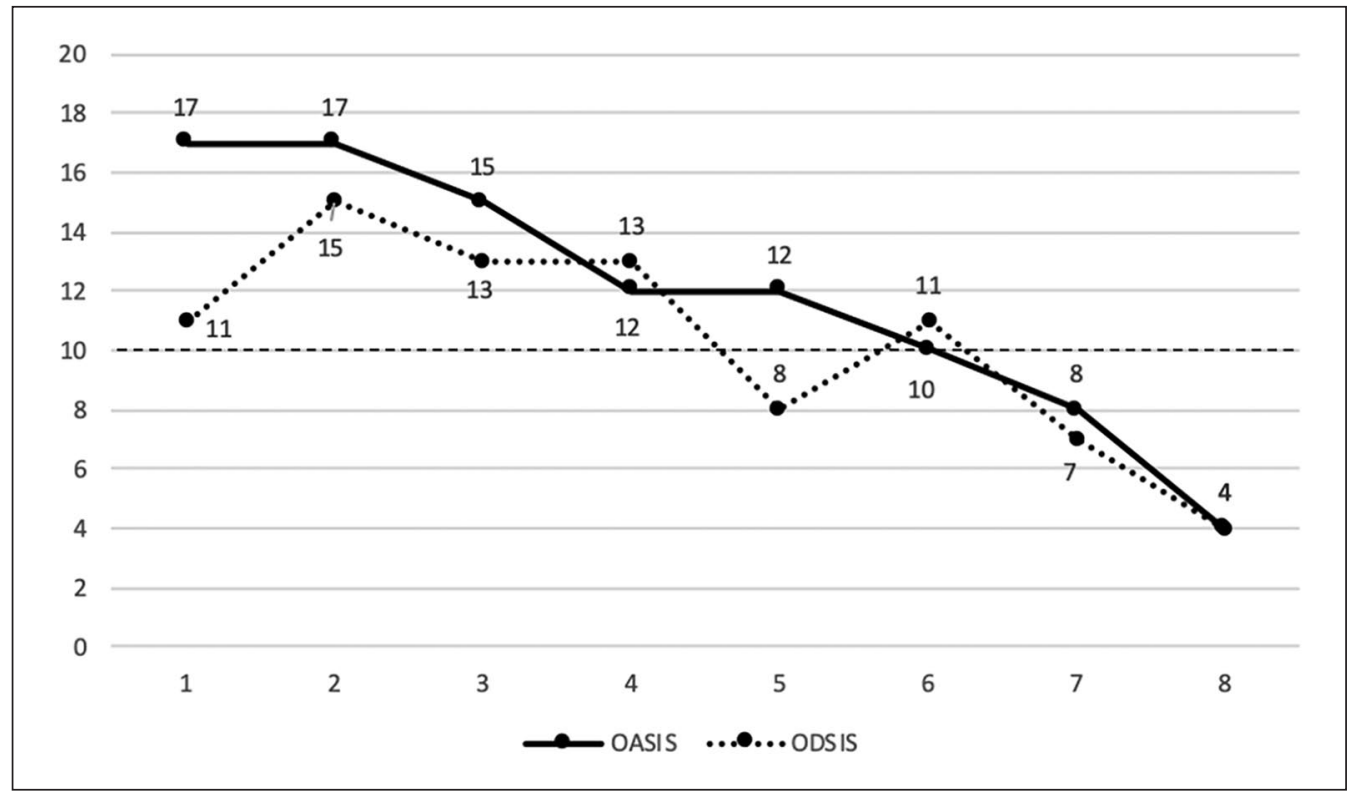

Figure 2. OASIS and ODSIS results throughout the eight modules of treatment.

As for the ODSIS, although scores decreased, "peaks" were observed in different modules. At the beginning of the intervention (Module 1), the ODSIS score was 11 points out of a maximum of 20. An increase in scores was observed in Module 2, coinciding with a family problem, with a total score of 15. Again, in Module 6, we found a slight increase in depressive symptomatology coinciding with a couple argument. However, in the last section of the figure, we can see how the depressive symptoms progressively decreased until reaching a total of 4 points in Module 8 of the intervention.

Both anxiety and depression scores at the posttest were below the cut-off point established for the Spanish clinical population, which was 10 points (dashed line in Figure 2).

Pretest and posttest changes in clinical variables: In addition to analyzing Patricia's evolution in anxiety and depression measures, we performed an analysis of the results before (pretest) and after treatment (posttest) in other clinical variables, namely PANAS-PA, PANAS-NA, and ASI-3.

As shown in Table 2, PANAS-PA increased significantly from pretest (15 out of 50 points) to posttest (32 out of 50 points). This change was clinically significant (Reliable Change Index $[\mathrm{RCI}]=5.48, p<.050)$. Conversely, PANAS-NA decreased significantly from a score of 29 at pretest to 12 at posttest $(\mathrm{RCI}=-5.31, p<.050)$. The initial ASI-3 score of 36 revealed high 
Table 2. Normative Data, Raw Scores, the Reliable Change Index of the Measures at the Pretest, Posttest, and 12-Month Follow-Up.

\begin{tabular}{|c|c|c|c|c|c|c|}
\hline & Normative data & Pre-test & Post-test & Follow-up & & \\
\hline & $M(S D)$ & RS & RS (PCP) & RS (PCP) & $\mathrm{RCl}$ & IN \\
\hline OASIS & 10.45 (4.49) & 17 & $4(76.47)$ & $6(64.7 I)$ & $-4.80^{\mathrm{a}}$ & SC, improvement. \\
\hline ODSIS & $9.88(5.14)$ & 11 & $4(63.64)$ & $5(54.54)$ & $-3.37^{a}$ & SC, improvement. \\
\hline EPDS & $5.73(4.29)$ & 13 & $3(76.92)$ & $4(69.23)$ & $-3.83^{\mathrm{a}}$ & SC, improvement. \\
\hline PANAS-PA & 30.37 (6.08) & 15 & $32(13.33)$ & & $5.48^{b}$ & SC, improvement. \\
\hline PANAS-NA & $22.69(6.83)$ & 29 & $12(58.62)$ & & $-5.3 I^{b}$ & SC, improvement. \\
\hline ASI-3 & $14.7(9.9)$ & 36 & $15(58.33)$ & & $-5.0^{\mathrm{b}}$ & SC, improvement. \\
\hline
\end{tabular}

Note. $M=$ mean; $S D=$ standard deviation; $\mathrm{RS}=$ raw score; $\mathrm{PCP}=$ percentage of change from pretest; $\mathrm{RCl}=$ Reliable Change Index; IN = interpretation; SC = significant change; OASIS = The Overall Anxiety Severity and Impairment Scale; ODSIS = The Overall Depression Severity and Impairment Scale; EPDS = Edinburg Postnatal Depression Scale; PANAS-PA = Positive and Negative Affect Schedule-Positive Affect; PANAS-NA= Positive and Negative Affect Schedule-Negative Affect; ASI-3 = Anxiety Sensitivity Index.

${ }^{a} \mathrm{RCl}$ was calculated comparing scores from pretest to follow-up.

${ }^{b} \mathrm{RCl}$ was calculated comparing scores from pretest to posttest.

sensitivity to anxiety, whereas, at posttest, the score was 15 . This change between pretest and posttest in sensitivity to anxiety was statistically significant $(\mathrm{RCI}=-5.00, p<.050)$.

The MINI interview was also administered at the end of the intervention, without Patricia meeting the criteria for any of the psychological disturbances. This indicates that the UP was effective in treating the anxious and depressive symptoms.

The qualitative evaluation of the patient after treatment was very positive, given that after completing it, she considered that she had "more resources and strategies to face day-to-day situations" and that it cost her "less to make decisions."

\section{Complicating Factors}

Several factors complicated our case. First, given Patricia's perinatal stage when she asked for help, she delivered in the middle of treatment. Secondly, she lived $1 \mathrm{hr}$ away from our clinic, so she could not physically come to our clinic with her child and the newborn. And third, besides MDD, she also presented obsessive personality traits, which led her to have more difficulties to make decisions. Given these obstacles, adaptations were made in the contents and structure of the UP for Patricia's specific case: she continued the treatment online after giving birth, and the Problem Solving Technique (D’Zurilla \& Nezu, 2007) was included to help her make decisions.

\section{Access and Barriers to Care}

Perinatal depression is an under-diagnosed and under-treated problem (Cox et al., 2016). Among the most common barriers to accessing mental health care resources are the stigma associated with mental illness, travel difficulties for patients, lack of time, physical limitations, and little knowledge about mental health issues and when to see a professional (Byatt et al., 2012; Maxfield $\&$ Segal, 2008). Therefore, recent research has suggested that the use of Information and Communication Technologies (ICTs; Internet, wireless networks, phones, mobile applications), with their advantage of anonymity, could be of great help in programs to assess and treat depressive symptoms in the perinatal stage (Donker et al., 2015). Moreover, there is evidence indicating that perinatal women and the general population both accept ICTs for mental health intervention programs (Miralles et al., 2020; Osma et al., 2020). 
In Patricia's case, difficulties to attend a consultation with her baby and child arose after she gave birth. Since email, videoconferencing, and telephone have emerged as important tools in providing online therapy (Roman et al., 2020), as of Module 4 (cognitive flexibility), the sessions were conducted via videoconference, after signing the appropriate consents. Support between sessions was provided by email and telephone.

\section{0 Follow-Up}

\section{Symptoms of Anxiety and Depression at the 12-Month Follow-Up}

Once the intervention was over, the posttest follow-up of the variables evaluated was carried out and subsequently, we contacted Patricia by telephone 6 months later to see how she was doing and, although no objective evaluation was carried out then, she did comment that she was generally well and that, except for a few odd days, she was controlling her emotions of anxiety and sadness. Finally, a follow-up was carried out after 12 months to verify that the results that had been achieved in the posttreatment evaluation were maintained over time without having to follow any other psychological or pharmacological treatment.

Figure 3 shows the results of the 12-month follow-up for the anxiety (OASIS) and depression (ODSIS and EPDS) variables. At the pretest, posttest, and 12-month follow-up assessments, in addition to the OASIS and ODSIS, the EPDS was administered as a specific instrument to assess depressive symptoms in the perinatal period. All three measures showed a slight increase in the 12-month follow-up scores compared to posttest. However, as shown in Table 2, these were significantly lower than the pretest scores for OASIS (RCI $=-4.80, p<.050)$, ODSIS (RCI $=-3.37$, $p<.050)$ and EPDS (RCI $=-3.83, p<.050)$. Similarly, the posttest score of EPDS was less than 11, a cut-off point established to consider clinical symptoms (Garcia-Esteve et al., 2003).

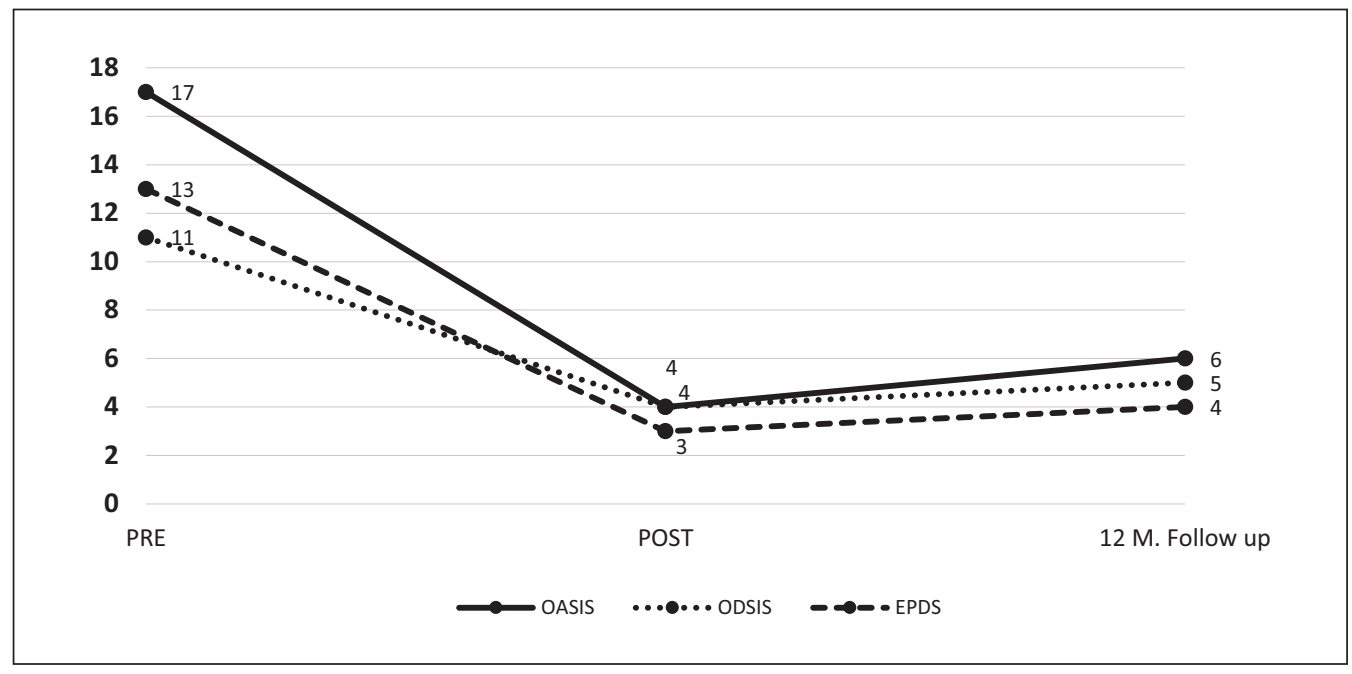

Figure 3. Results of clinical variables at the 12-month follow-up.

\section{I Treatment Implications of the Case}

In our study, we proposed the UP as being capable of reducing depressive and anxious symptoms in a 38-year-old pregnant woman. However, to include this emotion-regulation intervention in health systems serving perinatal women, we need to demonstrate its effectiveness. The UP, based on shared temperamental factors (Sherman et al., 2018), has been shown to be a valid 
intervention for the treatment of emotional disturbance in previous studies (Barlow et al., 2017). In this case study, the UP was effective in a case of MDD with peripartum onset, obsessive traits, and unspecified anxiety symptoms. The symptomatology and interference of anxiety and depression were reduced after the application of the UP. In the posttest evaluation, Patricia no longer met the diagnostic criteria for MDD, both because of subclinical scores on the posttest questionnaires (OASIS, ODSIS, and EPDS) and the MINI diagnostic interview at the 12-month followup. Therefore, we can propose the clinical utility and viability of the UP for the psychological treatment of perinatal depression.

The results of this study are important because the UP can be used when various intense emotions/symptoms (anxiety, sadness, guilt, anger, shame, etc.) or comorbid diagnoses are present, which is common in EDs (Barlow et al., 2013; Dennis et al., 2017), including peripartum-onset MDD. Among the many advantages of the UP, it is worth pointing out that clinical psychologists and psychologists in training can count on a structured and versatile intervention that focuses on the shared etiological and maintenance mechanisms of various EDs. Through the UP, we can work on the problems of emotional dysregulation shared by people with EDs, or in a subclinical or preventive way. This intervention simplifies the education and training of therapists and facilitates the dissemination of evidence-based transdiagnostic treatments (Barlow et al., 2004).

Another advantage of the UP is that it is a flexible treatment that allows the modules to be adapted and tailored to the objectives and needs of each patient (Sauer-Zavala et al., 2019). As stated in previous research, therapists must be able to address personal and contextual factors when following manualized protocols (Donahue et al., 2019), for example, by personalizing and adapting the modules, contents, and exercises. In the case of Patricia, several modifications were made, such as ending the face-to-face intervention and continuing via internet as soon as she gave birth, to make it easier for her to continue with the treatment. As we know, any psychotherapy can be delivered through online or offline format, and many studies have demonstrated psychotherapy effectiveness regardless of the format (García-Escalera et al., 2016) and, in our case, the UP has also proven to be effective in reducing anxious and depressive symptoms regardless of the format. In addition, treatment rapport and adherence, a key aspect of psychological interventions (Donahue et al., 2019), were qualitatively assessed with Patricia (e.g., How do you feel talking about these personal things of your life with me?) and they were maintained throughout the treatment after delivery via internet and during the follow-up. Also, adherence was evaluated taking into account the number of programmed appointments with the therapist and the attendance or cancellation of these sessions. No cancellation sessions occurred, so adherence was maintained in the online format. These data show that, as has been found in previous research, the use of ICTs in the area of perinatal mental health could be well accepted not only by health professionals but also by women in the perinatal stage (Martínez-Borba et al., 2019). We therefore consider that it is feasible to promote the use of the UP through ICTs in perinatal mental health. In this way, we would save time, effort, human and economic resources, which are especially relevant in rural contexts such as Patricia's (Harris \& Norton, 2018). Another option to deliver depression treatments in the patient's natural context is through in-home sessions (Maxfield \& Segal, 2008). In this sense, Ammerman et al. (2007) found that cognitive behavior therapy for a depressed mother delivered through home visitations resulted in a reduction of depressive symptoms, so we could anticipate that the UP protocol delivered in an in-home format could also result in a reduction of depressive and anxiety symptoms.

\section{2 Recommendations to Clinicians and Students}

Our results highlight the relevance of addressing women's mental health during the perinatal stage. Specifically, this care should begin in the first trimester of pregnancy and be maintained in 
the postpartum period (Byatt et al., 2018). In Patricia's case, depressive symptoms emerged in the 20th week of pregnancy and, far from receding during the following weeks of pregnancy, worsened as anxiety symptoms appeared. This underlines the importance of early attention to these symptoms to prevent their chronification. A paradoxical aspect that we found in this research is that, although the clinical recommendations advise evaluation and intervention at the beginning of pregnancy, most studies focus on the postpartum. As a solution, future research should target the evaluation and treatment of clinical symptoms at the beginning of pregnancy, and also considering the associated risk factors (Byatt et al., 2018).

This case study is the first step to propose future research studies, with larger samples and controlled experimental designs. Within the future lines, taking into account that the UP is effective in the treatment not only of depression but of other EDs, the target population could be expanded to include pregnant women with other diagnoses (e.g., adaptive disorder, eating disorder, obsessive and compulsive disorder, or other anxiety problems such as agoraphobia) that are usually addressed with specific treatment protocols (e.g., Christian \& Storch, 2009; Cullinan \& Gaynor, 2015). The UP could even be used as a prevention program for women who do not have any diagnosed problem, as has already been proposed in schools with adolescents (GarcíaEscalera et al., 2017). In fact, it would suffice to include it within the birth preparation programs, making the necessary adaptations to focus on emotion-regulation strategies and not so much on specific modules for clinical population.

Furthermore, thanks to the flexibility of the UP, intervention programs for women in the perinatal stage could reinforce some techniques included in the UP or include specific modules for women with additional stressors, for example, those in whom pregnancy was unwanted, unplanned, or women with a low economic income. Literature has shown that risk factors for the development of perinatal depression include unwanted and/or unplanned pregnancy and economic hardship (Norhayati et al., 2015). Therefore, these women could benefit from specific modules of acceptance, organization of material resources (e.g., income, job search) and personal resources (e.g., time management), or reinforce mindfulness modules to improve bonding (e.g., meditation exercises focused on the baby's movements or baby care). Previous research has applied techniques such as mindfulness to improve bonding with the baby, with positive results (Hicks et al., 2018). Working on maternal-fetal or maternal-infant bonding may be especially relevant for women whose pregnancy was unwanted/unplanned, and techniques included in the UP, such as mindfulness, may be beneficial in these cases.

As the UP has already been shown to be effective in a group format in clinical populations (Laposa et al., 2017), our next efforts should be aimed at applying the UP to women with perinatal depression/anxiety in a group format. Thus, we would take advantage of one of the main benefits of this type of format, that is, social support and vicarious learning from other women and mothers (Norton \& Hope, 2008) and, therefore, normalize the nature of their anxious/depressive symptoms.

In psychological work with perinatal women, working with partners or other family members is especially important (Pilkington et al., 2015). In Patricia's case, one of the objectives focused on improving her communication skills with her husband. This highlights the importance of including partners in interventions for perinatal depression, as has been seen in previous research. Variables such as marital adjustment or the women's perception of support are of enormous importance in maintaining the problem ( $\mathrm{Li}$ et al., 2019) so the partner should be included in maternal mental health care programs.

To our knowledge, this is the first study to analyze the clinical utility of the UP in a case of MDD with onset in the peripartum and comorbidity with anxiety symptoms. Despite the proven efficacy of the usual treatments for perinatal depression (e.g., cognitive-behavioral, interpersonal, or social support therapy), there are some barriers to their implementation. Although the present study is limited, as it is a case study with only one participant, the results indicate that these barriers could be solved with the application of transdiagnostic interventions such as the 
UP. This intervention has proven to be useful, flexible, and well accepted in the treatment of anxiety and depression symptoms. Finally, future lines of research should focus on (a) developing UP-based programs with onset during pregnancy; (b) considering the inclusion of the UP in prevention programs for perinatal women, preferably in a group or ICT-based format; (c) testing the suitability of including or developing specific modules for maternity and postpartum; and (d) including the partner or family members in the interventions.

\section{Acknowledgments}

We would like to thank every pregnant woman diagnosed with peripartum depression who was encouraged to ask for professional help, and especially the participant in this study who gave her permission for this publication.

\section{Declaration of Conflicting Interests}

The author(s) declared no potential conflicts of interest with respect to the research, authorship, and/or publication of this article.

\section{Funding}

The author(s) disclosed receipt of the following financial support for the research, authorship, and/or publication of this article: Gobierno de Aragón and FEDER 2014-2020 "Construyendo Europa desde Aragón" (S31_20D). Universitat Jaume I (PREDOC/2018/43).

\section{References}

American Psychiatric Association. (2013). Diagnostic and statistical manual of mental disorders (DSM-5). American Psychiatric Press.

Ammerman, R. T., Bodley, A. L., Putnam, F. W., Lopez, W. L., Holleb, L. J., Stevens, J., \& Van Ginkel, J. B. (2007). In-home cognitive behavior therapy for a depressed mother in a home visitation program. Clinical Case Studies, 6(2), 161-180. https://doi.org/10.1177/1534650106286533

Barlow, D., Allen, L., \& Choate, M. (2004). Toward a unified treatment for emotional disorders. Behavior Therapy, 35, 205-230.

Barlow, D. H., Farchione, T. J., Bullis, J. R., Gallagher, M. W., Murray-Latin, H., Sauer-Zavala, S., Bentley, K. H., Thompson-Hollands, J., Conklin, L. R., Boswell, J. F., Ametaj, A., Carl, J. R., Boettcher, H. T., \& Cassiello-Robbins, C. (2017). The unified protocol for transdiagnostic treatment of emotional disorders compared with diagnosis-specific protocols for anxiety disorders: A randomized clinical trial. JAMA Psychiatry, 74(9), 875-884. https://doi.org/10.1001/jamapsychiatry.2017.2164

Barlow, D. H., Sauer-Zavala, S., Carl, J. R., Bullis, J. R., \& Ellard, K. K. (2013). The nature, diagnosis, and treatment of neuroticism: Back to the future. Clinical Psychological Science, 2(3), 344-365. https://doi. org $/ 10.1177 / 2167702613505532$

Barlow, D., Sauer-Zavala, S., Farchione, T., Murray, H., Ellard, K., Bullis, J., \& Castellano-Robbins, C. (2018a). Protocolo unificado para el tratamiento transdiagnóstico de los trastornos emocionales (2nd ed.): Manual del terapeuta (Jorge Osma \& Elena Crespo, trad, 2019). Alianza Editorial.

Barlow, D., Sauer-Zavala, S., Farchione, T., Murray, H., Ellard, K., Bullis, J., \& Castellano-Robbins, C. (2018b). Protocolo unificado para el tratamiento transdiagnóstico de los trastornos emocionales (2nd ed.): Manual del paciente (Jorge Osma \& Elena Crespo, trad, 2019). Alianza Editorial.

Bentley, K., Gallagher, M., \& Barlow, D. (2014). Development and validation of the Overall Depression Severity and Impairment Scale. Psychological Assessment, 26(3), 815-830. https://doi.org/10.1037/ a0036216

Brown, T., Campbell, L., Lehman, C., Grisham, J., \& Mancill, R. (2001). Current and lifetime comorbidity of the DSM-IV anxiety and mood disorders in a large clinical sample. Journal of Abnormal Psychology, 110(4), 585-599.

Bullis, J. R., Boettcher, H., Sauer-Zavala, S., Farchione, T. J., \& Barlow, D. H. (2019). What is an emotional disorder? A transdiagnostic mechanistic definition with implications for assessment, treatment, and prevention. Clinical Psychology: Science and Practice, 26(2), e12278. https://doi.org/10.1111/cpsp.12278 
Byatt, N., Carter, D., Deligiannidis, K. M., Epperson, C. N., Meltzer-Brody, S., Payne, J. L., Robinson, G., Silver, N. E., Stowe, Z., Van Niel, M. S., Wisner, K. L., \& Yonkers, K. (2018). Position statement on screening and treatment of mood and anxiety disorders during pregnancy and postpartum. APA Official Actions.

Byatt, N., Simas, T. A. M., Lundquist, R. S., Johnson, J. V., \& Ziedonis, D. M. (2012). Strategies for improving perinatal depression treatment in North American outpatient obstetric settings. Journal of Psychosomatic Obstetrics and Gynecology, 33(4), 143-161. https://doi.org/10.3109/0167482X. 2012.728649

Caparros-Gonzalez, R. A., Perra, O., Alderdice, F., Lynn, F., Lobel, M., García-García, I., \& Peralta-Ramírez, M. I. (2019). Psychometric validation of the Prenatal Distress Questionnaire (PDQ) in pregnant women in Spain. Women and Health, 59(8), 937-952. https://doi.org/10.1080/03630242.2019.1584143

Christian, L. M., \& Storch, E. (2009). Clinical case studies cognitive behavioral treatment of aggressive obsessions. Clinical Case Studies, 8(1), 72-83. https://doi.org/10.1177/1534650108326974

Cox, E. Q., Sowa, N. A., Meltzer-Brody, S. E., \& Gaynes, B. N. (2016). The perinatal depression treatment cascade: Baby steps toward improving outcomes. The Journal of Clinical Psychiatry, 77(9), 1189-1200. https://doi.org/10.4088/JCP.15r10174

Cox, J. L., Holden, J. M., \& Sagovsky, R. (1987). Detection of postnatal depression: Development of the 10-item Edinburgh Postnatal Depression Scale. British Journal of Psychiatry, 150, 782-786. https:// doi.org/10.1192/bjp.150.6.782

Cullinan, C. C., \& Gaynor, S. T. (2015). Treatment of agoraphobia in a pregnant woman: A combination of exposure and acceptance and commitment therapy. Clinical Case Studies, 14(2), 98-114. https://doi. org/10.1177/1534650114539729

Danaher, B. G., Milgrom, J., Seeley, J. R., Stuart, S., Schembri, C., Tyler, M. S., Ericksen, J., Lester, W., Gemmill, A. W., Kosty, D. B., \& Lewinsohn, P. (2013). MomMoodBooster web-based intervention for postpartum depression: Feasibility trial results. Journal of Medical Internet Research, 15(11), e242. https://doi.org/10.2196/jmir.2876

Dennis, C. (2014). Psychosocial interventions for the treatment of perinatal depression. Best Practice \& Research Clinical Obstetrics \& Gynaecology, 28(1), 97-111. https://doi.org/10.1016/j.bpobgyn. 2013.08.008

Dennis, C., Brown, H. K., Wanigaratne, S., Fung, K., Vigod, S. N., Grigoriadis, S., Marini, F., \& Brennenstuhl, S. (2017). Prevalence, incidence, and persistence of postpartum depression, anxiety, and comorbidity among Chinese immigrant and nonimmigrant women: A longitudinal cohort study. The Canadian Journal of Psychiatry, 63(1), 44-53. https://doi.org/10.1177/0706743717720689

Donahue, J. M., Hormes, J. M., Gordis, E. B., \& Anderson, D. A. (2019). Attending to the alliance in the application of the unified protocol for the transdiagnostic treatment of emotional disorders: A case study. Clinical Case Studies, 18(4), 282-299. https://doi.org/10.1177/1534650119845503

Donker, T., Cuijpers, P., Stanley, D., \& Danaher, B. (2015). The future of perinatal depression identification: Can information and communication technology optimize effectiveness? In J. Milgrom \& A. Gemmill (Eds.), Identifying perinatal depression and anxiety. Evidence-based practise in screening psychosocial assessment, and management (pp. 240-255). Wiley-Blackwell.

D’Zurilla, T., \& Nezu, A. (2007). Problem-solving therapy: A positive approach to clinical intervention (3rd ed.). Springer.

García-Escalera, J., Chorot, P., Valiente, R. M., Reales, J. M., \& Sandín, B. (2016). Efficacy of transdiagnostic cognitive-behavioral therapy for anxiety and depression in adults, children and adolescents: A meta-analysis. Revista de Psicopatología y Psicología Clínica, 21, 147-175. https://doi.org/10.5944/ rppc.vol.21.num.3.2016.17811

García-Escalera, J., Valiente, R. M., Chorot, P., Ehrenreich-May, J., Kennedy, S. M., \& Sandín, B. (2017). The Spanish version of the unified protocol for transdiagnostic treatment of emotional disorders in adolescents (UP-A) adapted as a school-based anxiety and depression prevention program: Study protocol for a cluster randomized controlled trial. JMIR Research Protocols, 6(8), e149. https://doi.org/10.2196/ resprot.7934

Garcia-Esteve, L., Ascaso, C., Ojuel, J., \& Navarro, P. (2003). Validation of the Edinburgh Postnatal Depression Scale (EPDS) in Spanish mothers. Journal of Affective Disorders, 75(1), 71-76. https://doi. org/10.1016/S0165-0327(02)00020-4 
Hamel, C., Lang, E., Morissette, K., Beck, A., Stevens, A., Skidmore, B., Colquhoun, H., LeBlanc, J., Moore, A., Riva, J. J., Thombs, B. D., Colman, I., Grigoriadis, S., Nicholls, S. G., Potter, B. K., Ritchie, K., Robert, J., Vasa, P., Lauria-Horner, B., . . . Moher, D. (2019). Screening for depression in women during pregnancy or the first year postpartum and in the general adult population: A protocol for two systematic reviews to update a guideline of the Canadian Task Force on Preventive Health Care. Systematic Reviews, 8(1), 27.

Harris, K. R., \& Norton, P. J. (2018). Transdiagnostic cognitive behavioral therapy for the treatment of emotional disorders: A group case study. Clinical Case Studies, 17(6), 387-405. https://doi.org/ $10.1177 / 1534650118793938$

Hicks, L. M., Dayton, C. J., Brown, S., Muzik, M., \& Raveau, H. (2018). Mindfulness moderates depression and quality of prenatal attachment in expectant parents. Mindfulness, 9(5), 1604-1614. https://doi. org/10.1007/s12671-018-0907-2

Laposa, J., Mancuso, E., Abraham, G., \& Loli-Dano, L. (2017). Unified protocol transdiagnostic treatment in group format: A preliminary investigation with anxious individuals. Behavior Modification, 41(2), $253-268$.

Li, T., Guo, N., Jiang, H., Eldadah, M., \& Zhuang, W. (2019). Social support and second trimester depression. Midwifery, 69, 158-162. https://doi.org/10.1016/J.MIDW.2018.11.012

Lobel, M., \& Dunkel-Schetter, C. (2016). Pregnancy and prenatal stress. In H. Friedman (Ed.), Encyclopedia of mental health (pp. 318-329). Academic Press.

Martínez-Borba, V., Suso-ribera, C., \& Osma, J. (2019). Usability, acceptability, and feasibility of two technology-based devices for mental health screening in perinatal care: A comparison of web versus app. In P. Cipresso, S. Serino, \& D. Villani (Eds.), Pervasive computing paradigms for mental health (pp. 176-189). https://doi.org/10.1007/978-3-319-74935-8

Maxfield, M., \& Segal, D. L. (2008). Psychotherapy in nontraditional settings: A case of in-home cognitive-behavioral therapy with a depressed older adult. Clinical Case Studies, 7(2), 154-166. https://doi. org $/ 10.1177 / 1534650107307477$

McManus, F., Shafran, R., \& Cooper, Z. (2010). What does a transdiagnostic approach have to offer the treatment of anxiety disorders? British Journal of Clinical Psychology, 49, 491-505.

Milgrom, J., Holt, C., Holt, C. J., Ross, J., Ericksen, J., \& Gemmill, A. W. (2015). Feasibility study and pilot randomised trial of an antenatal depression treatment with infant follow-up. Archives of Women's Mental Health, 18(5), 717-730. https://doi.org/10.1007/s00737-015-0512-5

Miralles, I., Granell, C., García-Palacios, A., Castilla, D., González-Pérez, A., Casteleyn, S., \& BretónLópez, J. (2020). Enhancing in vivo exposure in the treatment of panic disorder and agoraphobia using location-based technologies: A case study. Clinical Case Studies, 19(2), 145-159. https://doi. org/10.1177/1534650119892900

Norhayati, M. N., Nik Hazlina, N. H., Asrenee, A. R., \& Wan Emilin, W. M. A. (2015). Magnitude and risk factors for postpartum symptoms: A literature review. Journal of Affective Disorders, 175, 34-52. https://doi.org/10.1016/j.jad.2014.12.041

Norman, S. B., Hami Cissell, S., Means-Christensen, A. J., \& Stein, M. B. (2006). Development and validation of an Overall Anxiety Severity And Impairment Scale (OASIS). Depression and Anxiety, 23(4), 245-249. https://doi.org/10.1002/da.20182

Norton, P. J., \& Hope, D. A. (2008). The "anxiety treatment protocol": A group case study demonstration of a transdiagnostic group cognitive-behavioral therapy for anxiety disorders. Clinical Case Studies, 7(6), 538-554. https://doi.org/10.1177/1534650108321307

Osma, J., Quilez-Orden, A., Suso-Ribera, C., Peris-Baquero, O., Norman, S., Bentley, K., \& Sauer-Zavala, S. (2019). Psychometric properties and validation of the Spanish versions of the Overall Anxiety and Depression Severity and Impairment Scales. Journal of Affective Disorders, 252, 9-18. https://doi. org/10.1016/j.jad.2019.03.063

Osma, J., Suso-Ribera, C., Martínez-Borba, V., \& Barrera, A. Z. (2020). Content and format preferences of a depression prevention program: A study in perinatal women. Anales de Psicologia, 36(1), 56-63. https://doi.org/10.6018/analesps.356051

Pilkington, P. D., Milne, L. C., Cairns, K. E., Lewis, J., \& Whelan, T. A. (2015). Modifiable partner factors associated with perinatal depression and anxiety: A systematic review and meta-analysis. Journal of Affective Disorders, 178, 165-180. https://doi.org/10.1016/j.jad.2015.02.023 
Roman, M., Constantin, T., \& Bostan, C. M. (2020). The efficiency of online cognitive-behavioral therapy for postpartum depressive symptomatology: A systematic review and meta-analysis. Women \& Health, 60(1), 99-112. https://doi.org/10.1080/03630242.2019.1610824

Sandín, B, Chorot, P., Lostao, L., Joiner, T. E., Santed, M. A., \& Valiente, R. M. (1999). Escalas PANAS de afecto positivo y negativo: Validacion factorial y convergencia transcultural. Psicothema, 11(1), 37-51. https://doi.org/ISSN 0214-9915

Sandín, B., Valiente, R. M., Chorot, P., \& Santed, M. (2007). ASI-3: Nueva escala para la evaluación de la sensibilidad a la ansiedad. Revista de Psicopatología y Psicología Clínica, 12(2), 91-104.

Sauer-Zavala, S., Cassiello-Robbins, C., Ametaj, A., Wilner, J., \& Pagan, D. (2019). Transdiagnostic treatment personalization: The feasibility of ordering unified protocol modules according to patient strengths and weaknesses. Behavior Modification, 43(4), 518-543.

Sheehan, D. (2015). Mini Neuropsychiatric International Interview 7.0 (MINI 7.0). Medical Outcomes Systems.

Sherman, J. A., Tonarely, N. A., \& Ehrenreich-May, J. (2018). Targeting comorbid anxiety and depression using the unified protocol for transdiagnostic treatment of emotional disorders in adolescents. Clinical Case Studies, 17(2), 59-76. https://doi.org/10.1177/1534650117753671

Sockol, L. E. (2018). A systematic review and meta-analysis of interpersonal psychoterapy for perinatal women. Journal of Affective Disorders, 232, 316-328. https://doi.org/10.1016/j.jad.2018.01.018

Taylor, S., Zvolensky, M. J., Cox, B. J., Deacon, B., Heimberg, R. G., Ledley, D. R., Abramowitz, J. S., Holaway, R. M., Sandin, B., Stewart, S. H., Coles, M., Eng, W., Daly, E. S., Arrindell, W. A., Bouvard, M., \& Cardenas, S. J. (2007). Robust dimensions of anxiety sensitivity: Development and initial validation of the Anxiety Sensitivity Index-3. Psychological Assessment, 19(2), 176-188. https:// doi.org/10.1037/1040-3590.19.2.176

Tsai, A. C., Scott, J. A., Hung, K. J., Zhu, J. Q., Matthews, L. T., Psaros, C., \& Tomlinson, M. (2013). Reliability and validity of instruments for assessing perinatal depression in African settings: Systematic review and meta-analysis. PLoS ONE, 8(12), e82511. https://doi.org/10.1371/journal. pone. 0082521

Watson, D., Clark, L. A., \& Tellegen, A. (1988). Development and validation of brief measures of positive and negative affect: The PANAS Scales. Journal of Personality and Social Psychology, 54(6), 1063. https://doi.org/10.1037/0022-3514.54.6.1063

World Health Organization. (2019). Maternal mental health. Author. Retrieved December 3, 2019, from https://www.who.int/mental_health/maternal-child/maternal_mental_health/en/

\section{Author Biographies}

Elena Crespo-Delgado is an assistant professor at Universitat Jaume I, Spain. Her global work interests lie in emotional regulation, perinatal mental health and personality disorders. She is particularly interested in transdiagnostic intervention of emotional disorders, prevention of perinatal mental disorders and treatment of borderline personality disorder.

Verónica Martínez-Borba is a PhD student at Universitat Jaume I, Spain. Her research interest is focused on perinatal mental health assessment, prevention and treatment as well as the use of ICTs in this field.

Jorge Osma is an associate professor at the University of Zaragoza. His research focuses on the prevention and treatment of emotional disorders using the Unified Protocol through cost-effective formats such as group therapy and ICTs. 